TRANSACTIONS OF THE

AMERICAN MATHEMATICAL SOCIETY

Volume 363, Number 12, December 2011, Pages 6465-6479

S 0002-9947(2011)05394-8

Article electronically published on July 26, 2011

\title{
THE SECOND MAIN THEOREM OF HOLOMORPHIC CURVES INTO PROJECTIVE SPACES
}

\author{
PEI-CHU HU AND CHUNG-CHUN YANG
}

\begin{abstract}
By utilizing Jacobian sections introduced by Stoll, we prove a second main theorem of holomorphic curves into projective spaces for hypersurfaces under certain conditions on the jets of the curves. The precise conditions are listed in three equations in the introduction. This solves a weaker form of Griffiths' conjecture and only for a special class of holomorphic curves.
\end{abstract}

\section{INTRODUCTION}

We will study a non-constant holomorphic curve

$$
f=\left[\mathfrak{f}_{0}, \ldots, \mathfrak{f}_{n}\right]: \mathbb{C} \longrightarrow \mathbb{P}^{n}=\mathbb{P}^{n}(\mathbb{C}),
$$

where $\mathfrak{f}_{0}, \ldots, \mathfrak{f}_{n}$ are entire functions on $\mathbb{C}$ without common zeros. Without loss of generality, we may assume $\mathfrak{f}_{0} \not \equiv 0$. Define

$$
\mathfrak{g}_{j}=\frac{\mathfrak{f}_{j}}{\mathfrak{f}_{0}}, j=1, \ldots, n .
$$

For any positive integer $k$, we obtain a holomorphic curve

$$
f_{k}=\left[1, \mathfrak{g}_{1}^{(k)}, \ldots, \mathfrak{g}_{n}^{(k)}\right]: \mathbb{C} \longrightarrow \mathbb{P}^{n}
$$

Let $z$ be the coordinate of $\mathbb{C}$ and let $f^{\prime}$ be the derivative of $f$ with respect to $z$ which is a mapping from $\mathbb{C}$ to the holomorphic tangent bundle $\mathbf{T}\left(\mathbb{P}^{n}\right)$ of $\mathbb{P}^{n}$. Let $H$ be the hyperplane section line bundle over the projective space $\mathbb{P}^{n}$. For a positive integer $d$, let $H^{d}$ be the $d$-th power of $H$. Let $\Gamma\left(\mathbb{P}^{n}, H^{d}\right)$ be the vector space of holomorphic sections of $H^{d}$ over $\mathbb{P}^{n}$. Now we state our main theorem:

Theorem 1.1. Take

$$
s_{j} \in \Gamma\left(\mathbb{P}^{n}, H^{d}\right)-\{0\}, j=1, \ldots, q,
$$

such that the divisor $D=D_{1}+\cdots+D_{q}$ has simple normal crossings in $\mathbb{P}^{n}$, where $D_{j}=\left(s_{j}=0\right)$ is the zero divisor of $s_{j}$. Let $f: \mathbb{C} \longrightarrow \mathbb{P}^{n}$ be a non-constant holomorphic curve such that

$$
f(\mathbb{C}) \nsubseteq \operatorname{supp}\left(D_{j}\right), f_{k}(\mathbb{C}) \nsubseteq \operatorname{supp}\left(D_{j}\right), j=1, \ldots, q, k=1, \ldots, n-1 .
$$

Received by the editors September 16, 2007 and, in revised form, December 17, 2009.

2010 Mathematics Subject Classification. Primary 32H02, 32H25.

Key words and phrases. Holomorphic curve, projective space, line bundle, Jacobian section, value distribution theory.

The work of the first author was partially supported by the Natural Science Foundation of China and Shandong.

The second author was partially supported by a UGC Grant of Hong Kong: Project No. 604103.

(C)2011 American Mathematical Society
Reverts to public domain 28 years from publication 6465 
Assume further that there exist homomorphisms

$$
t_{k}: f_{k}^{*}\left(\mathbf{T}\left(\mathbb{P}^{n}\right)\right) \longrightarrow f^{*}\left(\mathbf{T}\left(\mathbb{P}^{n}\right)\right), k=1, \ldots, n-1,
$$

such that lifted sections $f_{k, f_{k}}^{\prime}$ of $f_{k}^{\prime}$ for $f_{k}$ satisfy

$$
f_{f}^{\prime} \wedge t_{1}\left(f_{1, f_{1}}^{\prime}\right) \wedge \cdots \wedge t_{n-1}\left(f_{n-1, f_{n-1}}^{\prime}\right) \not \equiv 0 .
$$

Then for any $\varepsilon>0$,

$$
\begin{aligned}
\|(q d-n-1) T_{f}(r) & \leq \sum_{j=1}^{q} N_{f}\left(r, D_{j}\right)-N_{\operatorname{Ram}}(r, f) \\
& +\sum_{k=1}^{n-1} Y_{t_{k}}(r)+O\left(\log ^{+} T_{f}(r)\right)+O(\varepsilon \log r),
\end{aligned}
$$

where the symbol \| at the front of an inequality denotes that the inequality holds as $r \rightarrow \infty$ outside of a possible exceptional set $E$ with $\int_{E} d r / r<\infty$.

For the notation and symbols in Theorem [1.1, see Section 2 and (20). If we define the defect of $f$ for $D_{j}$,

$$
\delta_{f}\left(D_{j}\right)=1-\limsup _{r \rightarrow \infty} \frac{N_{f}\left(r, D_{j}\right)}{d T_{f}(r)},
$$

and if the homomorphisms (2) satisfy admissible conditions

$$
\|_{\varepsilon} \quad Y_{t_{k}}(r) \leq \varepsilon T_{f}(r), k=1, \ldots, n-1,
$$

for $\varepsilon>0$, then (4) immediately yields the defect relation

$$
\sum_{j=1}^{q} \delta_{f}\left(D_{j}\right) \leq \frac{n+1}{d} .
$$

P. Griffiths ([13], or cf. [23]) conjectured that (4) or (6]) holds if $f$ is algebraically non-degenerate. By the definition, a mapping $f: \mathbb{C} \longrightarrow \mathbb{P}^{n}$ is algebraically nondegenerate if the image of $f$ is not contained in any proper subvariety of $\mathbb{P}^{n}$. A. Biancofiore 1 proved (6) for a class of holomorphic curves. For holomorphic curves $f: \mathbb{C} \longrightarrow \mathbb{P}^{2}$, Siu 24 proved (6) with $n+1=3$ by using meromorphic connections. Theorem 1.1 mainly gives a special class of holomorphic curves satisfying (2), (3) and (5) such that (6) holds.

Special cases related to Theorem 1.1 and (6) , namely, $d=1$, were studied by Bloch [2, Cartan [4] and Dufresnoy [6, or see Fujimoto [7, 8], Green [9], 10], [11, Kobayashi 20, Lang [21, Wong-Wong [35, Wu 38, and Wang 34] for an analogue over function fields.

These results may be regarded as special cases of the following general problem:

Conjecture 1.2. Let $N$ be an algebraic manifold of dimension $n$ and let $D$ be a divisor on $N$ with normal crossings. There exists a proper algebraic subset $Z_{D}$ of $N$ having the following property. Let $f: \mathbb{C} \longrightarrow N$ be a holomorphic curve such that $f(\mathbb{C}) \nsubseteq Z_{D}$. Let $E$ be an ample divisor. Then

$$
\| \quad T_{f}(r,[D])+T_{f}\left(r, K_{N}\right) \leq N_{f}(r, D)+O\left\{\log \left(r T_{f}(r,[E])\right)\right\} .
$$


In this form the conjecture is due to S. Lang (cf. 21]). It includes the conjecture of P. Griffiths ([13] and [23]) as a special case. When $N$ is an Abelian variety, Siu and Yeung (cf. [27], 28]) solved Conjecture 1.2 in the affirmative. Generally, Siu (cf. 24, 25], 26], or 22]) gave some approaches by using meromorphic connections. See Vojta 32 for some new progress.

We note that the Griffiths and Lang conjectures have been translated into a general conjecture in Diophantine approximation by P. Vojta 31 and into a conjecture for holomorphic curves over non-Archimedean fields by $\mathrm{Hu}$ and Yang [18. Theorem 1.1 provides new supportive evidence for these conjectures.

\section{Preliminaries}

We briefly recall the concept of a Jacobian bundle of Jacobian sections first introduced and studied by W. Stoll (see 29] and 30]). Let $N$ be a smooth projective algebraic variety of dimension $n$. Take a positive integer $m$ with $n>m \geq 1$. Let $M$ be a complex manifold of dimension $m$ and consider a holomorphic mapping

$$
f: M \longrightarrow N \text {. }
$$

Let $\pi: E \longrightarrow N$ be a holomorphic vector bundle over $N$. The vector space of holomorphic sections of $E$ over $N$ is denoted by $\Gamma(N, E)$. The pullback bundle $\tilde{\pi}: f^{*}(E) \longrightarrow M$ is defined only up to an isomorphism such that $f \circ \tilde{\pi}=\pi \circ \tilde{f}$, where $\tilde{\pi}$ is the bundle projection and $\tilde{f}: f^{*}(E) \longrightarrow E$ is a bundle homomorphism over $f$. If $s \in \Gamma(N, E)$, then a lifted section $s_{f} \in \Gamma\left(M, f^{*}(E)\right)$ of $s$ for $f$ is uniquely defined by

$$
s_{f}(x)=\tilde{f}_{x}^{-1}(s(f(x))), \quad x \in M .
$$

Assume $s \not \equiv 0$ and $f(M) \not \subset s^{-1}(0)$. The lifted section $s_{f}$ exists with $s_{f} \not \equiv 0$.

Let $\mathbf{T}^{*}(N)$ be the dual of $\mathbf{T}(N)$. The canonical bundle of $N$ is defined by

$$
K_{N}=\bigwedge_{n} \mathbf{T}^{*}(N) \text {. }
$$

According to Stoll [29], the bundle

$$
K(f)=K_{M} \otimes f^{*}\left(K_{N}^{*}\right)
$$

is called the Jacobian bundle, where $K_{N}^{*}$ is the dual of $K_{N}$. A holomorphic section $F$ of $K(f)$ over $M$ is said to be a Jacobian section. The section $F$ is called effective if $F^{-1}(0)$ is thin. The zero divisor $(F)$ of $F$ is called the ramification divisor of $f$ for $F$.

Let $F$ be a Jacobian section and $U$ be an open subset in $N$ such that $\tilde{U}=$ $f^{-1}(U) \neq \emptyset$. The inner product

$$
\langle,\rangle: K_{N}^{*} \oplus K_{N} \longrightarrow \mathbb{C}
$$

is well defined and pulls back to

$$
\langle,\rangle: f^{*}\left(K_{N}^{*}\right) \oplus f^{*}\left(K_{N}\right) \longrightarrow \mathbb{C},
$$

which further induces a $K_{M}$-valued inner product

$$
\langle,\rangle: K(f) \oplus f^{*}\left(K_{N}\right) \longrightarrow K_{M} .
$$

The section $F$ defines a linear mapping

$$
F: \Gamma\left(U, K_{N}\right) \longrightarrow \Gamma\left(\tilde{U}, K_{M}\right)
$$


by $F[\psi]=\left\langle F, \psi_{f}\right\rangle$ for all $\psi \in \Gamma\left(U, K_{N}\right)$ and where $\psi_{f}$ is the lifted section defined previously. If $\left(V ; z_{1}, \ldots, z_{m}\right)$ and $\left(W ; w_{1}, \ldots, w_{n}\right)$ are holomorphic coordinate charts on $M$ and $N$ respectively with $W \subset U$ and $f(V) \subset W$, then $\psi=\psi_{W} d w$ on $W$ and $F=F_{V W} d z \otimes d^{*} w_{f}$ on $V$, where

$$
d w=d w_{1} \wedge \cdots \wedge d w_{n}, \quad d^{*} w=\frac{\partial}{\partial w_{1}} \wedge \cdots \wedge \frac{\partial}{\partial w_{n}},
$$

and where $\psi_{W}$ and $F_{V W}$ are holomorphic functions. Then on $V$ we have

$$
F[\psi]=F_{V W}\left(\psi_{W} \circ f\right) d z .
$$

Set

$$
i_{p}=\left(\frac{\sqrt{-1}}{2 \pi}\right)^{p}(-1)^{\frac{p(p+1)}{2}} p !
$$

Given a Jacobian section $F \in \Gamma(M, K(f))$, then a linear mapping

$$
F: A^{2 n}(U) \longrightarrow A^{2 m}(\tilde{U})
$$

is uniquely defined as follows: If $\Psi \in A^{2 n}(U)$ is expressed by

$$
\Psi=i_{n} \Psi_{W} d w \wedge \overline{d w}
$$

in terms of the holomorphic coordinate chart $\left(W ; w_{1}, \ldots, w_{n}\right)$ on $N$, then

$$
\left.F[\Psi]\right|_{\tilde{W}}=i_{m}\left(\Psi_{W} \circ f\right) F[d w] \wedge \overline{F[d w]} .
$$

Note that a homomorphism

$$
\hat{f}: f^{*}\left(\bigwedge_{m} \mathbf{T}^{*}(N)\right) \longrightarrow \bigwedge_{m} \mathbf{T}^{*}(M)
$$

exists uniquely such that $\hat{f}\left(\psi_{f}\right)=f^{*}(\psi)$ for all $\psi \in \Gamma\left(U, \bigwedge_{m} \mathbf{T}^{*}(N)\right)$, where $U$ is open in $N$ with $\tilde{U}=f^{-1}(U) \neq \emptyset$. Set $q=n-m$. The interior product

$$
\angle: K_{N} \oplus \bigwedge_{q} \mathbf{T}(N) \longrightarrow \bigwedge_{m} \mathbf{T}^{*}(N)
$$

also pulls back to an interior product

$$
\angle: f^{*}\left(K_{N}\right) \oplus f^{*}\left(\bigwedge_{q} \mathbf{T}(N)\right) \longrightarrow f^{*}\left(\bigwedge_{m} \mathbf{T}^{*}(N)\right) .
$$

Take a holomorphic section $\varphi \in \Gamma\left(M, f^{*}\left(\bigwedge_{q} \mathbf{T}(N)\right)\right)$, which is called a holomorphic field on $f$ over $M$ of degree $q$. Then $\varphi$ induces a Jacobian section $F_{\varphi}$ defined by

$$
\left.F_{\varphi}\right|_{\tilde{U}}=\hat{f}\left(\chi_{f} \angle \varphi\right) \otimes \chi_{f}^{*}
$$

where $\chi \in \Gamma\left(U, K_{N}\right)$ vanishes nowhere for the open subset of $N$ with $\tilde{U}=f^{-1}(U) \neq$ $\emptyset$ and $\chi^{*}$ is the dual frame. Equivalently, $F_{\varphi}$ can be described by its action

$$
F_{\varphi}[\psi]=\hat{f}\left(\psi_{f} \angle \varphi\right)
$$

for all $\psi \in \Gamma\left(U, K_{N}\right)$, where $U$ is open in $N$ with $\tilde{U}=f^{-1}(U) \neq \emptyset$. The section $\varphi$ is said to be effective for $f$ if $F_{\varphi}$ is effective.

Next we assume $M=\mathbb{C}^{m}$ with the standard holomorphic coordinates $z=$ $\left(z_{1}, \ldots, z_{m}\right)$. Define the exhaustion function $\tau: \mathbb{C}^{m} \longrightarrow[0,+\infty)$ by

$$
\tau(z)=\|z\|^{2}=\sum_{j=1}^{m}\left|z_{j}\right|^{2}
$$


and set

$$
v=d d^{c} \tau, \quad \sigma=d^{c} \log \tau \wedge\left(d d^{c} \log \tau\right)^{m-1},
$$

where

Take $r>0$. Define

$$
d^{c}=\frac{\sqrt{-1}}{4 \pi}(\bar{\partial}-\partial)
$$

$$
\mathbb{C}^{m}\langle 0 ; r\rangle=\left\{z \in \mathbb{C}^{m} \mid \tau(z)=r^{2}\right\}, \quad \mathbb{C}^{m}[0 ; r]=\left\{z \in \mathbb{C}^{m} \mid \tau(z) \leq r^{2}\right\} .
$$

If $h$ is an integrable function over $\mathbb{C}^{m}\langle 0 ; r\rangle$, we write

$$
\mathbb{C}^{m}\langle 0 ; r ; h\rangle=\int_{\mathbb{C}^{m}\langle 0 ; r\rangle} h \sigma .
$$

For an integrable $(1,1)$-form $\varphi$ on $\mathbb{C}^{m}$, set

$$
\mathbb{C}^{m}[0 ; r ; \varphi]=r^{2-2 m} \int_{\mathbb{C}^{m}[0 ; r]} \varphi \wedge v^{m-1}
$$

and define

$$
\mathcal{T}\left(r, r_{0} ; \varphi\right)=\int_{r_{0}}^{r} \frac{\mathbb{C}^{m}[0 ; t ; \varphi]}{t} d t
$$

for a fixed $r_{0}>0$ with $r>r_{0}$.

Let $\nu$ be a multiplicity on $\mathbb{C}^{m}$. For $t>0$, the counting function $n_{\nu}$ is defined by

$$
n_{\nu}(t)=t^{2-2 m} \int_{\mathbb{C}^{m}[0 ; t] \cap A} \nu v^{m-1},
$$

where $A=\operatorname{supp} \nu$. Here, if $m=1$, we define

$$
n_{\nu}(t)=\sum_{z \in \mathbb{C}[0 ; t] \cap A} \nu(z) .
$$

If $\nu$ is non-negative, then $n_{\nu}$ increases. The valence function of $\nu$ is defined by

$$
N_{\nu}(r)=N_{\nu}\left(r, r_{0}\right)=\int_{r_{0}}^{r} \frac{n_{\nu}(t)}{t} d t \quad\left(r \geq r_{0}\right) .
$$

Lemma 2.1 ([16], [19]). Define

$$
Z_{i}=f_{*}\left(\frac{\partial}{\partial z_{i}}\right), \quad i=1, \ldots, m .
$$

Then a holomorphic field $\varphi$ on $f$ over $\mathbb{C}^{m}$ of degree $n-m$ is such that $\varphi$ is effective for $f$ if and only if

$$
Z=Z_{1 f} \wedge \cdots \wedge Z_{m f} \wedge \varphi \not \equiv 0
$$

Further, we have $\left(F_{\varphi}=0\right)=(Z=0)$.

Lemma 2.2 ([16], [19]). Let $\varphi$ be an effective holomorphic field on $f$ over $\mathbb{C}^{m}$ of degree $n-m$. Define a non-negative function $g$ by

$$
F_{\varphi}\left[\psi^{n}\right]=g^{2} f^{*}\left(\psi^{m}\right),
$$

where $\psi$ is the associated 2-form of a Hermitian metric on $N$. Then $g \leq|\varphi|$, where $|\cdot|$ is the norm induced by the metric $\psi$. 
Lemma 2.3 ([15], 19]). Let $\eta \geq 0$ be a form of bidegree $(1,1)$ on $\mathbb{C}^{m}$ such that $\mathcal{T}\left(r, r_{0} ; \eta\right)$ exists for $r>r_{0}>0$. Let $u$ be a non-negative function on $\mathbb{C}^{m}$ satisfying

$$
u v^{m} \leq \eta \wedge v^{m-1} \text {. }
$$

Then for any $\varepsilon>0$,

$$
\| \quad \mathbb{C}^{m}\langle 0 ; r ; \log u\rangle \leq(1+2 \varepsilon) \log \mathcal{T}\left(r, r_{0} ; \eta\right)+4 \varepsilon \log r .
$$

Let $L$ be a line bundle over $N$. Take $0 \neq s_{j} \in \Gamma(N, L), j=1, \ldots, q$, such that the divisor $D=D_{1}+\cdots+D_{q}$ has normal crossings in $N$, where $D_{j}=\left(s_{j}=0\right)$ is irreducible. Assume that each $D_{j}(1 \leq j \leq q)$ is smooth, i.e., $D$ has simple normal crossings, and a positive number $\lambda$ exists such that Chern classes of $L$ and $K_{N}$ in de Rham cohomology $H_{\mathrm{DR}}^{2}(N, \mathbb{R})$ satisfy

$$
\lambda q c_{1}(L)+c_{1}\left(K_{N}\right)>0 .
$$

Obviously, such $\lambda$ exist if $L$ is positive. Then there are a volume form $\Omega$ on $N$ and a metric $\kappa$ of $L$ such that

$$
\begin{gathered}
0 \leq\left|s_{j}\right|_{\kappa}^{2}<\frac{1}{e}, \quad j=1, \ldots, q, \\
\lambda q c_{1}(L, \kappa)+\operatorname{Ric}(\Omega)>0
\end{gathered}
$$

and such that the form

$$
\Psi=\frac{\Omega}{\prod_{j=1}^{q}\left|s_{j}\right|_{\kappa}^{2 \lambda}\left(\log \left|s_{j}\right|_{\kappa}^{2}\right)^{2}},
$$

called a Carlson-Griffiths form, satisfies

$$
\operatorname{Ric}(\Psi)>0, \quad \operatorname{Ric}(\Psi)^{n} \geq \frac{\Omega}{\prod_{j=1}^{q}\left|s_{j}\right|_{\kappa}^{2}\left(\log \left|s_{j}\right|_{\kappa}^{2}\right)^{2}}, \quad \int_{N} \operatorname{Ric}(\Psi)^{n}<\infty ;
$$

see Carlson-Griffiths [3], Griffiths-King [14] and Stoll [29].

Lemma 2.4 ([3], 14], 29]). Let $L, D=D_{1}+\cdots+D_{q}, \lambda$ and $\Psi$ be as defined above. Assume that condition (12) holds. Let $f: \mathbb{C}^{m} \longrightarrow N$ be a holomorphic mapping satisfying the condition $f\left(\mathbb{C}^{m}\right) \nsubseteq \operatorname{supp}\left(D_{j}\right)$ for each $j=1, \ldots, q$. Then there exist a constant $c_{1} \leq 0$ such that for $r>r_{0}$,

$$
c_{1} \leq \lambda q T_{f}(r, L)+T_{f}\left(r, K_{N}\right)-\mathcal{T}\left(r, r_{0} ; f^{*}(\operatorname{Ric}(\Psi))\right) \leq q \log T_{f}(r, L)+O(1),
$$

where

$$
T_{f}(r, L)=\mathcal{T}\left(r, r_{0} ; f^{*}\left(c_{1}(L, \kappa)\right)\right), T_{f}\left(r, K_{N}\right)=\mathcal{T}\left(r, r_{0} ; f^{*}(\operatorname{Ric}(\Omega))\right)
$$

are well defined, up to $O(1)$.

Theorem 2.5 ([16]). Assume that $N$ is a compact complex manifold of dimension $n$. Let $L$ be a positive holomorphic line bundle on $N$. Take $0 \neq s_{j} \in \Gamma(N, L), j=$ $1, \ldots, q$, such that the divisor $D=D_{1}+\cdots+D_{q}$ has simple normal crossings in $N$, where $D_{j}=\left(s_{j}=0\right)$ is irreducible for each $j$. Assume that a positive number $\lambda$ exists such that Chern classes of $L$ and $K_{N}$ in de Rham cohomology $H_{\mathrm{DR}}^{2}(N, \mathbb{R})$ satisfy (11). Let $f: \mathbb{C}^{m} \longrightarrow N$ be a holomorphic mapping such that $f\left(\mathbb{C}^{m}\right) \nsubseteq s_{j}^{-1}(0)$ for all $j$. Assume that there exists an effective Jacobian section $F$ of $f$. Let $g$ be the function defined by

$$
F\left[\operatorname{Ric}(\Psi)^{n}\right]=g^{2} f^{*}\left(\operatorname{Ric}(\Psi)^{m}\right)
$$


where $\Psi$ is the Carlson-Griffiths form defined in (14). Then for any $\varepsilon>0$,

$$
\begin{aligned}
\| q T_{f}(r, L)+T_{f}\left(r, K_{N}\right) \leq & \sum_{j=1}^{q} N_{f}\left(r, D_{j}\right)-N_{\mu_{(F)}}(r)+\mathbb{C}^{m}\langle 0 ; r ; \log g\rangle \\
& +O\left(\log ^{+} T_{f}(r, L)\right)+O(\varepsilon \log r),
\end{aligned}
$$

where $\mu_{(F)}$ is the associated multiplicity of the zero divisor $(F=0)$ of $F$ over $\mathbb{C}^{m}$ (see [29] or [17]), and where

$$
N_{f}\left(r, D_{j}\right)=N_{\mu_{f^{*} D_{j}}}(r), \log ^{+} T_{f}(r, L)=\log \max \left\{1, T_{f}(r, L)\right\} .
$$

For an effective Jacobian section $F$ of $f$, we write

$$
N_{\text {Ram }}(r, f)=N_{\mu_{(F)}}(r) .
$$

In terms of a local coordinate system $w_{1}, \ldots, w_{n}$ of $N$ we may write

$$
\operatorname{Ric}(\Psi)=\frac{\sqrt{-1}}{2 \pi} \sum_{\alpha, \beta} h_{\alpha \beta} d w_{\alpha} \wedge d \bar{w}_{\beta} .
$$

The singular form $\operatorname{Ric}(\Psi)$ is smooth outside the divisor $D$, and hence defines a smooth metric

$$
h_{\Psi}=2 \sum_{\alpha, \beta} h_{\alpha \beta} d w_{\alpha} d \bar{w}_{\beta}
$$

which has "singularity" on $D$.

Theorem 2.6 ([19]). If $F$ is an effective Jacobian section of $f$ and if $f\left(\mathbb{C}^{m}\right) \nsubseteq$ $B(L) \neq N$, then there exists a positive constant c such that

$$
\| \quad c T_{f}(r, L)<\mathbb{C}^{m}\langle 0 ; r ; \log g\rangle+O(\varepsilon \log r)
$$

holds for any $\varepsilon>0$, where $g$ is defined by

$$
F\left[c_{1}(L, \kappa)^{n}\right]=g^{2} f^{*}\left(c_{1}(L, \kappa)^{m}\right) .
$$

\section{Proof of Theorem 1.1}

Now we show that Theorem 1.1 follows from the result:

Theorem 3.1. Take

$$
s_{j} \in \Gamma\left(\mathbb{P}^{n}, H^{d}\right)-\{0\}, j=1, \ldots, q,
$$

such that the divisor $D=D_{1}+\cdots+D_{q}$ has simple normal crossings in $\mathbb{P}^{n}$, where $D_{j}=\left(s_{j}\right)$ is the zero divisor of $s_{j}$. Let $f: \mathbb{C} \longrightarrow \mathbb{P}^{n}$ be a non-constant holomorphic curve such that

$$
f(\mathbb{C}) \nsubseteq \operatorname{supp}\left(D_{j}\right), j=1, \ldots, q .
$$

Assume that there exist holomorphic curves $f_{k}: \mathbb{C} \longrightarrow \mathbb{P}^{n}$ with

$$
f_{k}(\mathbb{C}) \nsubseteq \operatorname{supp}\left(D_{j}\right), j=1, \ldots, q, k=1, \ldots, n-1,
$$

and homomorphisms

$$
t_{k}: f_{k}^{*}\left(\mathbf{T}\left(\mathbb{P}^{n}\right)\right) \longrightarrow f^{*}\left(\mathbf{T}\left(\mathbb{P}^{n}\right)\right), k=1, \ldots, n-1,
$$

satisfying

$$
f_{f}^{\prime} \wedge t_{1}\left(f_{1, f_{1}}^{\prime}\right) \wedge \cdots \wedge t_{n-1}\left(f_{n-1, f_{n-1}}^{\prime}\right) \not \equiv 0 .
$$


Then for any $\varepsilon>0$,

$$
\| \quad \begin{aligned}
\| q d-n-1) T_{f}(r) & \leq \sum_{j=1}^{q} N_{f}\left(r, D_{j}\right)-N_{\operatorname{Ram}}(r, f)+\sum_{k=1}^{n-1} Y_{t_{k}}(r) \\
& +\left(\frac{1}{2}+\varepsilon\right) \sum_{k=1}^{n-1} \log T_{f_{k}}(r)+O\left(\log ^{+} T_{f}(r)\right)+O(\varepsilon \log r) .
\end{aligned}
$$

Proof. We will apply Theorem 2.5 to prove Theorem 3.1. To do this, we take

$$
m=1, N=\mathbb{P}^{n}, L=H^{d},
$$

and abbreviate

$$
T_{f}(r)=T_{f}(r, H) .
$$

By assumption, the holomorphic field

$$
\varphi=t_{1}\left(f_{1, f_{1}}^{\prime}\right) \wedge \cdots \wedge t_{n-1}\left(f_{n-1, f_{n-1}}^{\prime}\right)
$$

is effective for $f$. We get, via Lemma 2.1, an effective Jacobian section $F_{\varphi}$ of $f$. According to Theorem 2.5, a non-negative function $g$ is defined by

$$
F_{\varphi}\left[\psi^{n}\right]=g^{2} f^{*}(\psi)
$$

where $\psi=\operatorname{Ric}(\Psi)$. By Lemma 2.2, we have the estimate $g \leq|\varphi|$, where $|\varphi|$ is measured by the induced metric from $h_{\Psi}$.

It is easy to show that there exists a constant $c>0$ such that (cf. 19, Lemma $1.55)$

Setting

$$
|\varphi| \leq c\left|t_{1}\left(f_{1, f_{1}}^{\prime}\right)\right| \cdots\left|t_{n-1}\left(f_{n-1, f_{n-1}}^{\prime}\right)\right| .
$$

we obtain

$$
\mathbb{C}\langle 0 ; r ; \log g\rangle \leq \mathbb{C}\langle 0 ; r ; \log |\varphi|\rangle \leq \sum_{k=1}^{n-1} Y_{t_{k}}(r)+\frac{1}{2} \sum_{k=1}^{n-1} \mathbb{C}\left\langle 0 ; r ; \log \left|f_{k}^{\prime}\right|^{2}\right\rangle+O(1) .
$$

Further, Lemma 2.3 implies

$$
Y_{t_{k}}(r)=\mathbb{C}\left\langle 0 ; r ; \log \frac{\left|t_{k}\left(f_{k, f_{k}}^{\prime}\right)\right|}{\left|f_{k}^{\prime}\right|}\right\rangle
$$

$$
\| \quad \mathbb{C}\left\langle 0 ; r ; \log \left|f_{k}^{\prime}\right|^{2}\right\rangle \leq(1+2 \varepsilon) \log \mathcal{T}\left(r, r_{0} ;\left|f_{k}^{\prime}\right|^{2} v\right)+4 \varepsilon \log r .
$$

Note that

$$
\mathcal{T}\left(r, r_{0} ;\left|f_{k}^{\prime}\right|^{2} v\right)=\mathcal{T}\left(r, r_{0} ; f_{k}^{*}(\psi)\right) .
$$

By Lemma 2.4, there exist constants $c_{1} \leq 0, c_{2} \geq 0, c_{3} \geq 0$ such that for $r>r_{0}$,

$$
\begin{aligned}
c_{1} & \leq \lambda q T_{f_{k}}(r, L)+T_{f_{k}}\left(r, K_{N}\right)-\mathcal{T}\left(r, r_{0} ; f_{k}^{*}(\psi)\right) \\
& \leq q \log \left\{T_{f_{k}}(r, L)+c_{2}\right\}+c_{3} .
\end{aligned}
$$

Since $L=H^{d}, N=\mathbb{P}^{n}$, we have

$$
T_{f_{k}}(r, L)=d T_{f_{k}}(r, H)=d T_{f_{k}}(r), T_{f_{k}}\left(r, K_{N}\right)=-(n+1) T_{f_{k}}(r) .
$$

Then we obtain

$$
\mathcal{T}\left(r, r_{0} ; f_{k}^{*}(\psi)\right) \leq(\lambda q d-n-1) T_{f_{k}}(r)-c_{1} .
$$


Therefore

$$
\| \quad \mathbb{C}\langle 0 ; r ; \log g\rangle \leq \sum_{k=1}^{n-1} Y_{t_{k}}(r)+\left(\frac{1}{2}+\varepsilon\right) \sum_{k=1}^{n-1} \log T_{f_{k}}(r)+2(n-1) \varepsilon \log r+O(1),
$$

and so (18) follows from Theorem 2.5

To prove Theorem 1.1, we need to estimate the characteristic functions $T_{f_{k}}(r)$ of holomorphic curves (11). Note that $\mathfrak{f}_{0}^{k+1} \mathfrak{g}_{j}^{(k)}$ are entire functions such that $f_{k}$ can be expressed as follows:

$$
f_{k}=\left[\mathfrak{f}_{0}^{k+1}, \mathfrak{f}_{0}^{k+1} \mathfrak{g}_{1}^{(k)}, \ldots, \mathfrak{f}_{0}^{k+1} \mathfrak{g}_{n}^{(k)}\right] .
$$

Applying a formula due to Stoll (cf. [30], (6.63), or see [19], (2.3.20)),

$$
T_{f_{k}}(r)=\frac{1}{2} \mathbb{C}\left\langle 0 ; r ; \log \left|\mathfrak{f}_{0}^{k+1}\right|^{2}\left(1+\sum_{j=1}^{n}\left|\mathfrak{g}_{j}^{(k)}\right|^{2}\right)\right\rangle-N_{\nu_{k}}(r)+O(1),
$$

where $\nu_{k}$ is the multiplicity of common zeros of $\mathfrak{f}_{0}^{k+1}, \mathfrak{f}_{0}^{k+1} \mathfrak{g}_{1}^{(k)}, \ldots, \mathfrak{f}_{0}^{k+1} \mathfrak{g}_{n}^{(k)}$, we easily obtain the inequality

$$
\begin{aligned}
T_{f_{k}}(r) & \leq \frac{1}{2} \mathbb{C}\left\langle 0 ; r ; \log \left|\mathfrak{f}_{0}^{k+1}\right|^{2}\left(1+\left|\mathfrak{g}_{1}^{(k)}\right|^{2}+\cdots+\left|\mathfrak{g}_{n}^{(k)}\right|^{2}\right)\right\rangle+O(1) \\
& \leq(k+1) T_{f}(r)+\frac{1}{2} \mathbb{C}\left\langle 0 ; r ; \log \left(1+\left|\frac{\mathfrak{g}_{1}^{(k)}}{\mathfrak{g}_{1}}\right|^{2}+\cdots+\left|\frac{\mathfrak{g}_{n}^{(k)}}{\mathfrak{g}_{n}}\right|^{2}\right)\right\rangle+O(1) .
\end{aligned}
$$

Take $0<\alpha<1 /(2 k)$. We have

$$
\begin{aligned}
\frac{1}{2} \mathbb{C}\left\langle 0 ; r ; \log \left(1+\sum_{j}\left|\frac{\mathfrak{g}_{j}^{(k)}}{\mathfrak{g}_{j}}\right|^{2}\right)\right\rangle & =\frac{1}{\alpha} \mathbb{C}\left\langle 0 ; r ; \log \left(1+\sum_{j}\left|\frac{\mathfrak{g}_{j}^{(k)}}{\mathfrak{g}_{j}}\right|^{2}\right)^{\frac{\alpha}{2}}\right\rangle \\
& \leq \frac{1}{\alpha} \log \left\{1+\sum_{j} \mathbb{C}\left\langle 0 ; r ;\left|\frac{\mathfrak{g}_{j}^{(k)}}{\mathfrak{g}_{j}}\right|^{\alpha}\right\rangle\right\}
\end{aligned}
$$

Note that for any $\alpha$ with $0<\alpha<\frac{1}{2 k}$, the lemma of logarithmic derivative (cf. [39] or [19], Lemma 2.47) implies that there is a constant $C>1$ such that for any $r_{0}<r<\rho<R$,

$$
\mathbb{C}\left\langle 0 ; r ;\left|\frac{\mathfrak{g}_{j}^{(k)}}{\mathfrak{g}_{j}}\right|^{\alpha}\right\rangle \leq C\left\{\frac{\rho T\left(R, \mathfrak{g}_{j}\right)}{r(\rho-r)}\right\}^{k \alpha}, j=1, \ldots, n,
$$

where $T\left(R, \mathfrak{g}_{j}\right)$ is Nevanlinna's characteristic function of $\mathfrak{g}_{j}$. It is well known that (cf. [19], Lemma 2.37)

$$
T\left(R, \mathfrak{g}_{j}\right) \leq T_{f}(R)+O(1), j=1, \ldots, n .
$$

Therefore

$$
\frac{1}{2} \mathbb{C}\left\langle 0 ; r ; \log \left(1+\sum_{j}\left|\frac{\mathfrak{g}_{j}^{(k)}}{\mathfrak{g}_{j}}\right|^{2}\right)\right\rangle \leq k \log \left\{\frac{\rho T_{f}(R)}{r(\rho-r)}\right\}+O(1)
$$


for any $r_{0}<r<\rho<R$. Hence (cf. [19, (2.5.10), or [39])

$\| \frac{1}{2} \mathbb{C}\left\langle 0 ; r ; \log \left(1+\sum_{j}\left|\frac{\mathfrak{g}_{j}^{(k)}}{\mathfrak{g}_{j}}\right|^{2}\right)\right\rangle \leq k \log T_{f}(r)+k(1+\varepsilon) \log \log T_{f}(r)+O(1)$,

and so

$$
\| \quad T_{f_{k}}(r) \leq(k+1) T_{f}(r)+k \log T_{f}(r)+k(1+\varepsilon) \log \log T_{f}(r)+O(1) .
$$

Therefore

$$
\| \quad \mathbb{C}\langle 0 ; r ; \log g\rangle \leq \sum_{k=1}^{n-1} Y_{t_{k}}(r)+O\left(\log ^{+} T_{f}(r)\right)+O(\varepsilon \log r) .
$$

Thus Theorem 1.1 follows from Theorem 2.5 and (23).

\section{Notes}

We define $n+1$ holomorphic vector fields $Z_{0}, Z_{1}, \ldots, Z_{n}$ over $\mathbb{P}^{n}$ as follows. We consider the natural projection

$$
\mathbb{P}: \mathbb{C}^{n+1}-\{0\} \longrightarrow \mathbb{P}^{n}
$$

defined by $\left(\xi_{0}, \ldots, \xi_{n}\right) \mapsto\left[\xi_{0}, \ldots, \xi_{n}\right]$, which induces the holomorphic differential

$$
\mathbb{P}^{\prime}: \mathbf{T}\left(\mathbb{C}^{n+1}-\{0\}\right) \longrightarrow \mathbf{T}\left(\mathbb{P}^{n}\right) .
$$

Define

$$
Z_{i}=\mathbb{P}^{\prime}\left(\frac{\partial}{\partial \xi_{i}}\right), i=0,1, \ldots, n .
$$

Then $Z_{0}, \ldots, Z_{n}$ span the holomorphic tangent space at every point of $\mathbb{P}^{n}$. For example, denoting the local coordinates

$$
z_{i}=\frac{\xi_{i}}{\xi_{0}}, i=1, \ldots, n
$$

on the domain $U_{0}=\left\{\xi_{0} \neq 0\right\}$ of $\mathbb{P}^{n}(\mathbb{C})$, we have

$$
Z_{i}= \begin{cases}\frac{1}{\xi_{0}} \partial_{i}, & \text { if } 1 \leq i \leq n \\ -\sum_{j=1}^{n} \frac{\xi_{j}}{\xi_{0}^{2}} \partial_{j}, & \text { if } i=0\end{cases}
$$

where

$$
\partial_{i}=\frac{\partial}{\partial z_{i}}, i=1, \ldots, n
$$

Now the holomorphic curve

$$
f=\left[\mathfrak{f}_{0}, \ldots, \mathfrak{f}_{n}\right]: \mathbb{C} \longrightarrow \mathbb{P}^{n}
$$

satisfies

$$
f^{\prime}=\sum_{i=0}^{n} \mathfrak{f}_{i}^{\prime} Z_{i}
$$

and so

$$
f_{f}^{\prime}=\sum_{i=0}^{n} \mathfrak{f}_{i}^{\prime} Z_{i f} .
$$

Similarly, the associated holomorphic curves

$$
f_{k}=\left[\mathfrak{f}_{0}^{k+1}, \mathfrak{f}_{0}^{k+1} \mathfrak{g}_{1}^{(k)}, \ldots, \mathfrak{f}_{0}^{k+1} \mathfrak{g}_{n}^{(k)}\right]: \mathbb{C} \longrightarrow \mathbb{P}^{n}
$$


satisfy

$$
f_{k}^{\prime}=(k+1) \mathfrak{f}_{0}^{k} \mathfrak{f}_{0}^{\prime} Z_{0}+\sum_{i=1}^{n}\left(\mathfrak{f}_{0}^{k+1} \mathfrak{g}_{i}^{(k)}\right)^{\prime} Z_{i}
$$

with

Set

$$
f_{k, f_{k}}^{\prime}=(k+1) \mathfrak{f}_{0}^{k} \mathfrak{f}_{0}^{\prime} Z_{0 f_{k}}+\sum_{i=1}^{n}\left(\mathfrak{f}_{0}^{k+1} \mathfrak{g}_{i}^{(k)}\right)^{\prime} Z_{i f_{k}} .
$$

$$
t_{k}\left(Z_{i f_{k}}\right)=\sum_{j=0}^{n} a_{k i j} Z_{j f}
$$

We make further computations on the domain $U_{0}$, and find

$$
f^{\prime}=\sum_{i=1}^{n} \mathfrak{g}_{i}^{\prime} \partial_{i}, f_{k}^{\prime}=\sum_{i=1}^{n} \mathfrak{g}_{i}^{(k+1)} \partial_{i}
$$

and hence

Note that

$$
f_{f}^{\prime}=\sum_{i=1}^{n} \mathfrak{g}_{i}^{\prime} \partial_{i f}, f_{k, f_{k}}^{\prime}=\sum_{i=1}^{n} \mathfrak{g}_{i}^{(k+1)} \partial_{i f_{k}}
$$

$$
t_{k}\left(\partial_{i f_{k}}\right)=\sum_{j=1}^{n}\left(a_{k i j} f_{0}^{k}-a_{k i 0} \mathfrak{f}_{0}^{k-1} \mathfrak{f}_{j}\right) \partial_{j f}
$$

and so

$$
t_{k}\left(f_{k, f_{k}}^{\prime}\right)=\sum_{j=1}^{n} \sum_{i=1}^{n}\left(a_{k i j} \mathfrak{f}_{0}^{k}-a_{k i 0} \mathfrak{f}_{0}^{k-1} \mathfrak{f}_{j}\right) \mathfrak{g}_{i}^{(k+1)} \partial_{j f} .
$$

If we define

$$
\mathfrak{g}_{j k}= \begin{cases}\mathfrak{g}_{j}^{\prime}, & \text { if } k=0, \\ \sum_{i=1}^{n}\left(a_{k i j} \mathfrak{f}_{0}^{k}-a_{k i 0} \mathfrak{f}_{0}^{k-1} \mathfrak{f}_{j}\right) \mathfrak{g}_{i}^{(k+1)}, & \text { if } 1 \leq k \leq n-1,\end{cases}
$$

then the non-degeneracy condition (3) is just equivalent to

$$
\operatorname{det}\left(\mathfrak{g}_{j k}\right) \not \equiv 0 \text {. }
$$

Set $f_{0}=f$. Since every holomorphic vector bundle on $\mathbb{C}$ is trivial, we may choose holomorphic trivialization

$$
h_{k}: f_{k}^{*}\left(\mathbf{T}\left(\mathbb{P}^{n}\right)\right) \longrightarrow \mathbb{C}^{n+1}
$$

and construct the homomorphisms

$$
t_{k}=h_{0}^{-1} \circ h_{k}, k=1, \ldots, n-1 .
$$

Let $e_{1}, \ldots, e_{n}$ be the standard unit vectors of $\mathbb{C}^{n}$. If we can choose $e_{0} \in \mathbb{C}^{n}$ such that for each $k=0, \ldots, n-1$, there exists a holomorphic trivialization (25) satisfying

$$
h_{k}\left(Z_{j f_{k}}(z)\right)=\left(z, e_{j}\right), z \in \mathbb{C}, j=0,1, \ldots, n,
$$

we have

$$
t_{k}\left(\sum_{j=0}^{n} z_{j} Z_{j f_{k}}(z)\right)=\sum_{j=0}^{n} z_{j} Z_{j f}(z) .
$$

Thus by using the coordinates on $U_{0}$, we find

$$
\left|t_{k}\left(f_{k, f_{k}}^{\prime}\right)\right|=\sum_{\alpha, \beta} \mathfrak{g}_{\alpha}^{(k+1)} \overline{\mathfrak{g}}_{\beta}^{(k+1)} h_{\alpha \beta} \circ f,
$$


but

$$
\left|f_{k}^{\prime}\right|=\sum_{\alpha, \beta} \mathfrak{g}_{\alpha}^{(k+1)} \overline{\mathfrak{g}}_{\beta}^{(k+1)} h_{\alpha \beta} \circ f_{k} .
$$

Hence the key point estimating $Y_{t_{k}}(r)$ is how to compare $h_{\alpha \beta} \circ f_{k}$ with $h_{\alpha \beta} \circ f$ sharply.

Griffiths' conjecture would yield the following problem (cf. [10]):

Conjecture 4.1. Let $f: \mathbb{C} \longrightarrow \mathbb{P}^{n}$ be a holomorphic curve omitting a non-singular divisor without repeated components of degree at least $n+2$. Then the image of $f$ lies in a proper algebraic subvariety of $\mathbb{P}^{n}$.

For example, take a positive integer $d \geq 4$, let $P$ be a non-constant polynomial on $\mathbb{C}$ and consider the holomorphic curve

$$
f=\left[1, e^{P}, e^{\frac{d}{d-1} P}\right]: \mathbb{C} \longrightarrow \mathbb{P}^{2}
$$

Green [11] used this holomorphic curve to show that $f$ omits the divisor

$$
D=\left\{\xi_{0} \xi_{2}^{d-1}=\xi_{1}\left(\xi_{1}^{d-1}-\xi_{0}^{d-1}\right)\right\},
$$

but is contained in the hypersurface

$$
N=\left\{\xi_{1}^{d}=\xi_{0} \xi_{2}^{d-1}\right\} .
$$

It is easy to check that $f_{1}(\mathbb{C}) \nsubseteq D$. Theorem 1.1 now claims that there is no admissible homomorphism

$$
t_{1}: f_{1}^{*}\left(\mathbf{T}\left(\mathbb{P}^{2}\right)\right) \longrightarrow f^{*}\left(\mathbf{T}\left(\mathbb{P}^{2}\right)\right)
$$

satisfying

$$
f_{f}^{\prime} \wedge t_{1}\left(f_{1, f_{1}}^{\prime}\right) \not \equiv 0
$$

Finally, we give an application of jet bundles. Let $N$ be a smooth complex projective variety of dimension $n$, let $k$ be a positive integer, let $J_{k}(N)$ denote the holomorphic jet bundle of all $k$-jets of $N$ so that $J_{1}(N)$ is the holomorphic tangent bundle $\mathbf{T}(N)$ of $N$, let $f: \mathbb{C} \longrightarrow N$ be a holomorphic curve, and let $j_{k}(f): \mathbb{C} \longrightarrow J_{k}(N)$ denote the canonical lifting of $f$. According to the proof of Theorem 1.1. Theorem 2.5 then easily yields the following result:

Theorem 4.2. Let $N$ be a smooth complex projective variety of dimension $n$ and let $L$ be a positive line bundle over $N$. Take

$$
s_{j} \in \Gamma(N, L)-\{0\}, j=1, \ldots, q,
$$

such that the divisor $D=D_{1}+\cdots+D_{q}$ has simple normal crossings in $N$, where $D_{j}=\left(s_{j}=0\right)$ is the zero divisor of $s_{j}$. Let $f: \mathbb{C} \longrightarrow N$ be a non-constant holomorphic curve with $f(\mathbb{C}) \nsubseteq \operatorname{supp}(D)$ such that there exist holomorphic bundle mappings

$$
t_{k}: J_{k}(N) \longrightarrow \mathbf{T}(N), k=2, \ldots, n,
$$

satisfying

$$
f^{\prime} \wedge t_{2}\left(j_{2}(f)\right) \wedge \cdots \wedge t_{n}\left(j_{n}(f)\right) \not \equiv 0
$$


Then for any $\varepsilon>0$,

$$
\begin{aligned}
\| q T_{f}(r, L)+T_{f}\left(r, K_{N}\right) & \leq \sum_{j=1}^{q} N_{f}\left(r, D_{j}\right)+\sum_{k=2}^{n} \mathbb{C}\left\langle 0 ; r ; \log \left|t_{k}\left(j_{k}(f)\right)\right|\right\rangle \\
& -N_{\operatorname{Ram}}(r, f)+O\left(\log ^{+} T_{f}(r, L)\right)+O(\varepsilon \log r) .
\end{aligned}
$$

To estimate the terms $\mathbb{C}\left\langle 0 ; r ; \log \left|t_{k}\left(j_{k}(f)\right)\right|\right\rangle$ in (28), we need the lemma of logarithmic derivatives due to P. M. Wong [36] (or see [37, [5], 32], [33]):

Theorem 4.3. Let $N$ be a projective variety and let

(i) $D$ be an effective divisor with simple normal crossings or

(ii) $D$ be the trivial divisor in $N$ (i.e. the support of $D$ is empty or, equivalently, the line bundle associate to $D$ is trivial).

Let $f: \mathbb{C} \longrightarrow N$ be a holomorphic curve and let $L$ be any ample line bundle on $N$. Then there exists a singular jet metric $\rho_{k}$ on $J_{k}(N)(-\log D)$ satisfying

$$
\| \quad \mathbb{C}\left\langle 0 ; r ; \log ^{+} \rho_{k}\left(j_{k}(f)\right)\right\rangle \leq O\left(\log ^{+} T_{f}(r, L)\right)+O(\varepsilon \log r) .
$$

The metric $\rho_{k}$ is constructed as follows: Since there exists a finite number of rational functions $R_{1}, \ldots, R_{p}$ on $N$ such that the logarithmic jet differentials

$$
\left\{\left(\frac{d^{(j)} R_{i}}{R_{i}}\right)^{\frac{m}{j}} \mid 1 \leq i \leq p, 1 \leq j \leq k\right\}
$$

span the fibers of the sheaf $\mathcal{J}_{k}^{m}(N)(\log D)$ of $k$-jet differentials of weight $m$ over every point of $N$ (see [37, the proof of Theorem A1), the metric $\rho_{k}$ is defined by

$$
\rho_{k}(\xi)=\sum_{i=1}^{p} \sum_{j=1}^{k}\left|\left(\frac{d^{(j)} R_{i}}{R_{i}}\right)^{\frac{m}{j}}(\xi)\right|^{2}, \xi \in J_{k}(N)(-\log D) .
$$

Next we use Theorem 4.3 to the divisor $D$ of Theorem 4.2 , and further, assume that the holomorphic bundle mappings (26) decrease the metrics $\rho_{k}$, that is,

$$
\rho_{1}\left(t_{k}(\xi)\right) \leq \lambda_{k} \rho_{k}(\xi)^{\alpha_{k}}, \xi \in J_{k}(N), k=2, \ldots, n,
$$

for some positive constants $\lambda_{k}$ and $\alpha_{k}$. From the choice of $R_{i}$ and the express of $\operatorname{Ric}(\Psi)$ (see the proof of Lemma 6.21 in [19]), it is easy to find a constant $c^{\prime}$ such that

$$
h_{\Psi} \leq c^{\prime} \rho_{1}
$$

and hence

$$
\left|t_{k}\left(j_{k}(f)\right)\right| \leq c^{\prime} \rho_{1}\left(t_{k}\left(j_{k}(f)\right)\right), k=2, \ldots, n .
$$

Therefore,

$$
\left|t_{k}\left(j_{k}(f)\right)\right| \leq c^{\prime} \lambda_{k} \rho_{k}\left(j_{k}(f)\right)^{\alpha_{k}}, k=2, \ldots, n,
$$

since the bundle mappings $t_{k}$ decrease the metrics $\rho_{k}$. By using Theorem 4.3, we obtain

$$
\sum_{k=2}^{n} \mathbb{C}\left\langle 0 ; r ; \log \left|t_{k}\left(j_{k}(f)\right)\right|\right\rangle \leq O\left(\log ^{+} T_{f}(r, L)\right)+O(\varepsilon \log r),
$$


and hence (28) yields

$$
\begin{aligned}
\| q T_{f}(r, L)+T_{f}\left(r, K_{N}\right) & \leq \sum_{j=1}^{q} N_{f}\left(r, D_{j}\right)-N_{\operatorname{Ram}}(r, f) \\
& +O\left(\log ^{+} T_{f}(r, L)\right)+O(\varepsilon \log r) .
\end{aligned}
$$

\section{ACKNOWLEDGEMENT}

The authors want to thank the anonymous referee's many significant helpful remarks and suggestions.

\section{REFERENCES}

[1] Biancofiore, A., A hypersurface defect relation for a class of meromorphic maps, Trans. Amer. Math. Soc. 270 (1982), 47-60. MR642329 (83h:32005)

[2] Bloch, A., Sur les systèmes de fonctions holomorphes à variétés linéaires lacunaires, Ann. Ecole. Norm. Sup. 43 (1926), 309-362. MR.1509274

[3] Carlson, J. and Griffiths, P., Defect relation for equidimensional holomorphic mappings between algebraic varieties, Ann. of Math. 95 (1972), 557-584. MR.0311935 (47:497)

[4] Cartan, H., Sur les systèmes de fonctions holomorphes à variétés linéaires lacunaires, Ann. Sci. Ecole Norm. Sup. 45 (1928), 255-346. MR.1509288

[5] Chandler, K. and Wong, P. M., Finsler geometry of holomorphic jet bundles, in "A Sampler in Riemann/Finsler Geometry", MSRI Publ. 50, Cambridge University Press (2004), 107-196. MR2132659 (2006i:32022)

[6] Dufresnoy, H., Théorie nouvelle des familles complexes normales. Applications à l'étude des fonctions algébrö̈des, Ann. Sci. Ecole Norm. Sup. 61 (1944), 1-44. MR0014469 (7:289f)

[7] Fujimoto, H., Extensions of the big Picard theorem, Tohoku Math. J. 24 (1972), 415-422. MR0333253 (48:11578)

[8] Fujimoto, H., Families of holomorphic maps into the projective space omitting some hyperplanes, J. Math. Soc. Japan 25 (1973), 235-249. MR0328131 (48:6473)

[9] Green, M., Holomorphic maps into complex projective space omitting hyperplanes, Trans. Amer. Math. Soc. 169 (1972), 89-103. MR0308433 (46:7547)

[10] Green, M., Some Picard theorems for holomorphic mappings to algebraic varieties, Amer. J. Math. 97 (1975), 43-75. MR0367302 (51:3544)

[11] Green, M., Some examples and counter examples in value distribution theory of several complex variables, Composito Math. 39 (1975), 317-322. MR.0374498 (51:10698)

[12] Green, M. and Griffiths, P., Two applications of algebraic geometry to entire holomorphic mappings, The Chern Symposium 1979 (Proc. Internat. Sympos., Berkeley, Calif., 1979), Springer-Verlag, New York, 1980, pp. 41-74. MR609557(82h:32026)

[13] Griffiths, P., Holomorphic mappings: Survey of some results and discussion of open problems, Bull. Amer. Math. Soc. 78 (1972), 374-382. MR0294718 (45:3786)

[14] Griffiths, P. and King, J., Nevanlinna theory and holomorphic mappings between algebraic varieties, Acta Math. 130 (1973), 145-220. MR0427690 (55:721)

[15] Hu, P. C., Holomorphic mapping into algebraic varieties of general type, Nagoya Math. J. 120 (1990), 155-170. MR 1086576 (92b:32033)

[16] Hu, P. C., Holomorphic mappings between spaces of different dimensions I, Math. Z. 214 (1993), 567-577; II, Math. Z. 215 (1994), 187-193. MR1248113 (94k:32043) MR.1259455 (95a:32046)

[17] Hu, P. C., P. Li and Yang, C. C., Unicity of meromorphic mappings, Kluwer, 2003. MR:1988768 (2004m:32031)

[18] $\mathrm{Hu}$, P. C. and Yang, C. C., Meromorphic functions over non-Archimedean fields, Mathematics and Its Applications 522, Kluwer Academic Publishers, 2000. MR 1794326 (2002a:11085)

[19] $\mathrm{Hu}$, P. C. and Yang, C. C., Value distribution theory related to number theory, Birkhäuser, 2006. MR2245631(2008c:32021)

[20] Kobayashi, S., Hyperbolic complex spaces, GMW 318, Springer, 1998. MR1635983 (99m:32026) 
[21] Lang, S., Introduction to complex hyperbolic spaces, Springer-Verlag, 1987. MR886677 (88f:32065)

[22] Nadel, A. M., Hyperbolic surfaces in $\mathbb{P}^{3}$, Duke Math. J. 58 (1989), 749-771. MR 1016444 (91a:32036)

[23] Shiffman, B., Holomorphic curves in algebraic manifolds, Bull. Amer. Math. Soc. 83 (1977), 553-568. MR0440075 (55:12956)

[24] Siu, Y. T., Defect relations for holomorphic maps between spaces of different dimensions, Duke Math. J. 55 (1) (1987), 213-251. MR883671 (89a:32030)

[25] Siu, Y. T., Nonequidimensional value distribution theory and meromorphic connections, Duke Math. J. 61 (2) (1990), 341-367. MR1074301 (92f:32044)

[26] Siu, Y. T., Nonequidimensional value distribution theory, Lecture Notes in Math. 1351, 285311, Springer-Verlag, 1988. MR982094 (90j:32028)

[27] Siu, Y. T. and Yeung, S. K., Defects for ample divisors of Abelian varieties, Schwarz lemma, and hyperbolic hypersurfaces of low degrees, Amer. J. Math. 119 (1997), 1139-1172. MR:1473072 (98h:32044)

[28] Siu, Y. T. and Yeung, S. K., Addendum to "Defects for ample divisors of Abelian varieties, Schwarz lemma, and hyperbolic hypersurfaces of low degrees", Amer. J. Math. 125 (2003), 441-448. MR1980629 (2004e:32028)

[29] Stoll, W., Value distribution on parabolic spaces, Lecture Notes in Math. 600 (1977), SpringerVerlag. MR 0450626 (56:8919)

[30] Stoll, W., The Ahlfors-Weyl theory of meromorphic maps on parabolic manifolds, Lecture Notes in Math. 981 (1983), 101-219, Springer-Verlag. MR699135 (85c:32045)

[31] Vojta, P., Diophantine approximation and value distribution theory, Lecture Notes in Math. 1239, Springer, 1987. MR883451 (91k:11049)

[32] Vojta, P., On the ABC conjecture and Diophantine approximation by rational points, Amer. J. Math. 122 (2000), 843-872. MR1771576 (2001i:11094)

[33] Vojta, P., Correction to: "On the $A B C$ conjecture and Diophantine approximation by rational points" [Amer. J. Math. 122 (2000), no. 4, 843-872; MR.1771576 (2001i:11094)]. Amer. J. Math. 123 (2001), no. 2, 383-384. MR.1828227 (2002d:11095)

[34] Wang, J. T. Y., The truncated second main theorem of function fields, J. Number Theory 58 (1) (1996), 139-157. MR.1387732 (97c:11074)

[35] Wong, P. M. and Wong, Philip P. W., The second main theorem on generalized parabolic manifolds, preprint.

[36] Wong, P. M., Nevanlinna theory for holomorphic curves in projective varieties, preprint.

[37] Wong, P. M. and Stoll, W., On holomorphic jet bundles, math. AG/0003226, preprint.

[38] $\mathrm{Wu}, \mathrm{H}$. , The equidistribution theory of holomorphic curves, Princeton University Press, Princeton, New Jersey, 1970. MR0273070(42:7951)

[39] Ye, Z., On Nevanlinna's second main theorem in projective space, Invent. Math. 122 (1995), 475-507. MR1359601 (96j:32030)

Department of Mathematics, Shandong University, Jinan 250100, Shandong, People's Republic of China

E-mail address: pchu@sdu.edu.cn

Department of Mathematics, The Hong Kong University of Science \& Technology, Hong Kong, People's Republic of China

E-mail address: chungchun.yang@gmail.com 